\title{
Convex Optimization-based Controller Design for Stochastic Nonlinear Systems using Contraction Analysis
}

\author{
Hiroyasu Tsukamoto and Soon-Jo Chung
}

\begin{abstract}
This paper presents an optimal feedback tracking controller for a class of Itô stochastic nonlinear systems, the design of which involves recasting a nonlinear system equation into a convex combination of multiple non-unique State-Dependent Coefficient (SDC) models. Its feedback gain and controller parameters are found by solving a convex optimization problem to minimize an upper bound of the steady-state tracking error. Multiple SDC parametrizations are utilized to provide a design flexibility to mitigate the effects of stochastic noise and to ensure that the system is controllable. Incremental stability of this controller is studied using stochastic contraction analysis and it is proven that the controlled trajectory exponentially converges to the desired trajectory with a non-vanishing error due to the linear matrix inequality statedependent algebraic Riccati equation constraint. A discretetime version of stochastic contraction analysis with respect to a state- and time-dependent metric is also presented in this paper. A simulation is performed to show the superiority of the proposed optimal feedback controller compared to a known exponentially-stabilizing nonlinear controller and a PID controller.
\end{abstract}

\section{INTRODUCTION}

The problem of designing a controller for Itô stochastic nonlinear systems [1] is of significant importance in control theory as many engineering systems are nonlinear and have to deal with stochastic uncertainty to improve the controller performance. The probability density function of stochastic processes that are governed by Itô stochastic differential equations exhibits non-Gaussian behavior characterized by the Fokker-Plank equation [1], [2], which makes the controller design particularly difficult.

One approach to dealing with stochastic disturbances is to ignore or model them as deterministic disturbances in order to design a deterministic controller using feedback linearization [3]-[5] or Control Lyapunov Functions (CLFs) [5]-[7]. For a nonlinear system with a polynomial or rational vector field, the process of finding a CLF can be formulated as sum of squares programming [8], [9]. The State-Dependent Riccati Equation (SDRE) method [10], [11] can be used for systems that are written in SDC linear structure.

These controllers, however, could violate control requirements for stochastically perturbed systems. The proposed controller, in contrast, is based on the SDRE method and guarantees exponential stability of Itô stochastic nonlinear systems with some bounded error.

There are some controllers specifically designed for stochastic nonlinear systems as in this paper, one of which

The authors are with the Graduate Aerospace Laboratories (GALCIT), California Institute of Technology, 1200 E California Blvd, Pasadena, CA, USA. E-mail: \{htsukamoto, sjchung\}ecaltech.edu is a Lyapunov-like technique. This includes [12], which provides sufficient conditions for the existence of stabilizing feedback laws. In [13], a CLF-based controller design for stochastic nonlinear systems with unknown covariance is presented. A backstepping-based controller [14], [15] is shown to be asymptotically stabilizing in probability for a class of stochastic nonlinear systems in strict-feedback or output-feedback form. A controller design for Hamiltonian systems with stochastic disturbance is also well-studied [16]. An $H_{\infty}$ control problem for a class of stochastic nonlinear systems with both state- and disturbance-dependent noise is discussed in [17]. In the context of optimal control, the maximum principle for stochastic nonlinear optimal control problems in the general case is studied in [18]. In [19], a locally-optimal feedback control law for stochastic nonlinear systems is derived by applying a Linear Quadratic Gaussian (LQG) method iteratively to a linearized and discretized dynamic model. For stochastic dynamic programs [1], [20], some prior works have proposed different ways to overcome the issues arising from the curse of dimensionality [21], [22]. Stochastic Model Predictive Control (SMPC) [23], [24] has been derived to incorporate the probabilistic descriptions of uncertainties into a stochastic optimal control problem.

Unlike these controllers developed for stochastic nonlinear systems, the proposed feedback controller is different in that it can be used for any Itô stochastic nonlinear systems written in SDC form and also is optimal in a sense that it minimizes an upper bound of the steady-state tracking error in a stochastic manner. This choice of the objective function is more advantageous than integral objective functions as it allows us to write the controller gain synthesis algorithm as a convex optimization problem.

The tools to analyze incremental stability [25] of nonlinear systems have been developed in response to emerging needs in proving stability of trajectories instead of stability of an equilibrium point. Contraction analysis [26], [27] is one effective tool for studying incremental stability properties. The stochastic version of contraction analysis is derived in [28] with an application to the specialized context of state-independent metrics. In [29], the incremental stability of stochastic systems using more generalized metric functions that are both state- and time-dependent is studied. Contraction analysis for discrete-time and hybrid systems is provided in [26], [30], [31] and its stochastic counterpart is investigated in [32] with respect to a state-independent metric. In this paper, we describe more general discrete-time incremental contraction analysis with respect to a state- and time-dependent metric. 
The objective of this paper is to present a novel optimal tracking control approach to a class of Itô stochastic nonlinear systems with guaranteed exponential stability, which in turns results in a superior property of robustness, similar to finite-gain $L_{p}$ stability of a deterministic system. There are three major contributions of this paper.

1) A convex optimization-based algorithm to select the optimal feedback gain and controller parameters at each time instant is proposed in Sec. IV for the control of Itô stochastic nonlinear systems. The design is based on nonunique SDC forms and the control law is assumed to be a simple state feedback. The main idea of this algorithm lies in solving an optimization problem, the objective of which is to minimize an upper bound of the steady-state tracking error while satisfying the SDRE. As an attempt to avoid computational complexity in real-time operation, the original nonlinear optimization problem is reformulated as a convex optimization problem, with the SDRE constraint relaxed to an Linear Matrix Inequality (LMI) constraint with some additional constraints [33]. The feedback gain and controller parameters, including the coefficients of a convex combination of multiple SDC parameterizations are selected as a result of the convex optimization problem, which can be solved using various computationally-efficient numerical methods [33]-[35]. The superiority of this controller to the prior work [36] and to a PID controller is shown using results of a numerical simulation in Sec. V. Note that this controller design is a significant improvement over the observer design [29], whose optimization-cost function uses a linear combination of controller parameters without accounting for the contraction constraint.

2) Stability of the feedback tracking controller is studied using stochastic incremental contraction analysis with a stateand time-dependent Riemannian metric introduced in [29]. It is proved in Sec. III that the trajectory of the controlled dynamics exponentially converges to the desired trajectory with some known non-vanishing tracking error, which will be minimized by solving the convex optimization problem in Sec. IV. Multiple SDC parameterizations of the nonlinear system provide a controller design flexibility to mitigate the effect of stochastic noise while verifying that the system is controllable.

3) A discrete-time version of stochastic contraction analysis with respect to a state- and time-dependent metric is derived in Sec. II, which can be used for proving incremental stability of discrete-time and hybrid stochastic nonlinear systems, along with the known results for deterministic systems [30], [31].

\section{CONTRACTION ANALYSis}

In this section, we introduce some preliminaries that will be used for the stability analysis in Sec. III. We also present a new theorem for analyzing stochastic incremental stability of discrete-time stochastic systems with respect to a stateand time-dependent Riemannian metric.

\section{A. Continuous-time Dynamical Systems}

Consider the following continuous-time nonlinear system and its virtual dynamics

$$
\dot{x}=f(x, t), \quad \delta \dot{x}=\frac{\partial f(x, t)}{\partial x} \delta x .
$$

Lemma 1: The system (1) is contracting (i.e., all the solution trajectories exponentially converge to a single trajectory globally from any initial condition), if there exists a uniformly positive definite metric $M(x, t)=\Theta(x, t)^{T} \Theta(x, t)$, $M(x, t) \succeq \underline{m} I, \forall x, t$, with a smooth coordinate transformation of the virtual displacement $\delta z=\Theta(x, t) \delta x$ s.t.

$$
\frac{\partial f^{T}}{\partial x} M(x, t)+\dot{M}(x, t)+M(x, t) \frac{\partial f}{\partial x} \preceq-2 \gamma M(x, t), \forall x, t
$$

where $\gamma$ and $\underline{m}$ are some positive constants. If the system (1) is contracting, then the following equation holds

$$
\|\delta z(t)\|=\|\Theta(x, t) \delta x(t)\| \leq\|\delta z(0)\| e^{-\gamma t} .
$$

Proof: See [26], [37].

Next, consider the continuous-time nonlinear system (1) with a stochastic perturbation given by an Itô stochastic differential equation

$$
d x=f(x, t) d t+G(x, t) d W, x(0)=x_{0} .
$$

We assume that the following conditions for existence and uniqueness of a solution to (4) hold

$$
\begin{aligned}
& \exists L_{1}>0, \forall t, \forall x_{1}, x_{2} \in \mathbb{R}^{n} \text { s.t } \\
& \left\|f\left(x_{1}, t\right)-f\left(x_{2}, t\right)\right\|+\left\|G\left(x_{1}, t\right)-G\left(x_{2}, t\right)\right\|_{F} \leq L_{1}\left\|x_{1}-x_{2}\right\| \\
& \exists L_{2}>0, \forall t, \forall x_{1} \in \mathbb{R}^{n} \text { s.t } \\
& \left\|f\left(x_{1}, t\right)\right\|^{2}+\left\|G\left(x_{1}, t\right)\right\|_{F}^{2} \leq L_{2}\left(1+\left\|x_{1}\right\|^{2}\right)
\end{aligned}
$$

where $G: \mathbb{R}^{n} \times \mathbb{R} \rightarrow \mathbb{R}^{n \times d}$ is a matrix-valued function, $W(t)$ is a $d$-dimensional Wiener process, and $x_{0}$ is a random variable independent of $W(t)$. Now, consider the following two systems with trajectories $\xi_{1}(t)$ and $\xi_{2}(t)$ driven by two independent Wiener processes $W_{1}(t)$ and $W_{2}(t)$

$$
d \xi=\left[\begin{array}{l}
f\left(\xi_{1}, t\right) \\
f\left(\xi_{2}, t\right)
\end{array}\right] d t+\left[\begin{array}{cc}
G_{1}\left(\xi_{1}, t\right) & 0 \\
0 & G_{2}\left(\xi_{2}, t\right)
\end{array}\right]\left[\begin{array}{l}
d W_{1} \\
d W_{2}
\end{array}\right]
$$

where $\xi(t)=\left[\xi_{1}(t)^{T} \xi_{2}(t)^{T}\right]^{T} \in \mathbb{R}^{2 n}$. The following lemma [29] analyzes stochastic incremental stability of the two trajectories $\xi_{1}(t)$ and $\xi_{2}(t)$ with respect to each other in the presence of stochastic noise. The trajectories of (4) are parameterized as $x(0, t)=\xi_{1}$ and $x(1, t)=\xi_{2}$, and $G_{1}\left(\xi_{1}, t\right)$ and $G_{2}\left(\xi_{2}, t\right)$ are defined as $G(x(0, t), t)=G_{1}\left(\xi_{1}, t\right)$ and $G(x(1, t), t)=G_{2}\left(\xi_{2}, t\right)$.

Lemma 2: Assume that the system (6) has the following bounds, $\left.\operatorname{tr}\left(G_{i}\left(\xi_{i}, t\right)^{T} M\left(\xi_{i}, t\right) G_{i}\left(\xi_{i}, t\right)\right)\right) \leq C_{i}, \quad i=1,2$, $\bar{m}_{x}=\sup _{t \geq 0, i, j}\left\|\left(M_{i j}\right)_{x}\right\|$, and $\bar{m}_{x^{2}}=\sup _{t \geq 0, i, j}\left\|\partial^{2}\left(M_{i j}\right) / \partial x^{2}\right\|$, where $C_{1}, C_{2}, \bar{m}_{x}$, and $\bar{m}_{x^{2}}$ are constant. Assume also that (2) holds (i.e., contracting) for the deterministic system (1). Consider the generalized squared length with respect to a Riemannian metric $M(x(\mu, t), t)$ defined by $V(x, \delta x, t)=\int_{0}^{1}(\partial x / \partial \mu)^{T} M(x(\mu, t), t)(\partial x / \partial \mu) d \mu$ s.t. $V(x, \delta x, t) \geq \underline{m}\left\|\xi_{1}-\xi_{2}\right\|_{2}^{2}$. Then, the mean squared distance 
between the two trajectories of the system (6) whose initial conditions given by a probability distribution $p\left(a_{0}, b_{0}\right)$ are independent of $W_{1}(t)$ and $W_{2}(t)$ satisfies the bound

$E\left[\left\|\xi_{1}(t)-\xi_{2}(t)\right\|^{2}\right] \leq \frac{1}{\underline{m}}\left(\frac{C}{2 \gamma_{1}}+E[V(x(0), \delta x(0), 0)] e^{-2 \gamma t}\right)$

where $\gamma_{1}=\gamma-\left(\left(g_{1}^{2}+g_{2}^{2}\right) / 2 \underline{m}\right)\left(\varepsilon \bar{m}_{x}+\bar{m}_{x^{2}} / 2\right)>0, C=C_{1}+$ $C_{2}+\left(\bar{m}_{x} / \varepsilon\right)\left(g_{1}^{2}+g_{2}^{2}\right), \quad\left\|G_{1}\right\|_{F} \leq g_{1}, \quad \forall x, t$, and $\left\|G_{2}\right\|_{F} \leq$ $g_{2}, \forall x, t . E[\cdot]$ denotes the expected value of the operator and $\gamma$ is the contraction rate for the deterministic system (1).

Proof: See [29].

\section{B. Discrete-time Nonlinear Dynamical Systems}

We have a result similar to Lemma 1 for the following discrete-time nonlinear system and its virtual dynamics

$$
x_{k+1}=f_{k}\left(x_{k}, k\right), \quad \delta x_{k+1}=\frac{\partial f_{k}\left(x_{k}, k\right)}{\partial x_{k}} \delta x_{k} .
$$

Lemma 3: The system (8) is contracting if there exists a uniformly positive definite metric $M_{k}\left(x_{k}, k\right)=$ $\Theta_{k}\left(x_{k}, k\right)^{T} \Theta_{k}\left(x_{k}, k\right), M_{k}\left(x_{k}, k\right) \succeq \underline{m I}, \forall x_{k}, k$, with a smooth coordinate transformation of the virtual displacement $\delta z_{k}=$ $\Theta_{k}\left(x_{k}, k\right) \delta x_{k}$ s.t.

$$
\frac{\partial f_{k}^{T}}{\partial x_{k}} M_{k+1}\left(x_{k+1}, k+1\right) \frac{\partial f_{k}}{\partial x_{k}} \preceq\left(1-\gamma_{d}\right) M_{k}\left(x_{k}, k\right), \forall x_{k}, k
$$

where $\gamma_{d} \in(0,1)$ and $\underline{m}$ are some positive constants. If the system (8) is contracting, then the following equation holds

$$
\left\|\delta z_{k}\right\|=\left\|\Theta_{k}\left(x_{k}, k\right) \delta x_{k}\right\| \leq\left\|\delta z_{0}\right\|\left(1-\gamma_{d}\right)^{\frac{k}{2}}
$$

Proof: See [26], [31], [37].

We now present a discrete-time version of Lemma 2, which can be extensively used for proving the stability of discrete-time and hybrid stochastic nonlinear systems, along with the known results for deterministic systems [30], [31]. Consider the discrete-time nonlinear system (8) with a stochastic perturbation modeled by the stochastic difference equation

$$
x_{k+1}=f_{k}\left(x_{k}, k\right)+G_{k}\left(x_{k}, k\right) w_{k}
$$

where $G_{k}: \mathbb{R}^{n} \times \mathbb{R} \rightarrow \mathbb{R}^{n+d}$ is a matrix-valued function and $w_{k}$ is a $d$-dimensional sequence of zero mean uncorrelated normalized Gaussian random variables. Consider following two systems with trajectories $\xi_{1, k}$ and $\xi_{2, k}$ driven by two independent stochastic perturbation $w_{1, k}$ and $w_{2, k}$

$$
\xi_{k+1}=\left[\begin{array}{l}
f_{k}\left(\xi_{1, k}, k\right) \\
f_{k}\left(\xi_{2, k}, k\right)
\end{array}\right]+\left[\begin{array}{cc}
G_{1, k}\left(\xi_{1, k}, k\right) & 0 \\
0 & G_{2, k}\left(\xi_{2, k}, k\right)
\end{array}\right]\left[\begin{array}{l}
w_{1, k} \\
w_{2, k}
\end{array}\right]
$$

where $\xi_{k}=\left[\begin{array}{ll}\xi_{1, k}^{T} & \xi_{2, k}^{T}\end{array}\right]^{T} \in \mathbb{R}^{2 n}$. The following theorem analyzes stochastic incremental stability for discrete-time nonlinear systems. Theorem 1 is different from [38] in that the form of the Lyapunov function is assumed and its Riemannian metric is state- and time-dependent. The trajectories of (11) are parameterized as $x_{k}(\mu=0)=\xi_{1, k}, x_{k}(\mu=1)=\xi_{2, k}$, $w_{k}(\mu=0)=w_{1, k}, w_{k}(\mu=1)=w_{2, k}$, and $G_{1, k}\left(\xi_{1, k}, k\right)$ and
$G_{2, k}\left(\xi_{2, k}, k\right)$ are defined as $G_{k}\left(x_{k}(\mu=0), k\right)=G_{1, k}\left(\xi_{1, k}, k\right)$ and $G_{k}\left(x_{k}(\mu=1), k\right)=G_{2, k}\left(\xi_{2, k}, k\right)$.

Theorem 1: Assume that the system (12) has the following bounds, $M_{k}\left(x_{k}, k\right) \preceq \bar{m} I, \forall x_{k}, k$ and $\operatorname{Tr}\left(G_{1, k}^{T} G_{1, k}+\right.$ $\left.G_{2, k}^{T} G_{2, k}\right) \leq C_{d} / \bar{m}, \quad \forall \xi_{1, k}, \xi_{2, k}, k$, where $\bar{m}$ and $C_{d}$ are some positive constants and $G_{i, k}=G_{i, k}\left(\xi_{i, k}, k\right), \quad i=$ 1,2 for notation simplicity. Assume also that (9) holds for the discrete-time deterministic system (8). Consider the generalized squared length with respect to a Riemannian metric $M_{k}\left(x_{k}(\mu), k\right)$ defined by $v_{k}\left(x_{k}, \delta x_{k}, k\right)=$ $\int_{0}^{1}\left(\partial x_{k} / \partial \mu\right)^{T} M_{k}\left(x_{k}(\mu), k\right)\left(\partial x_{k} / \partial \mu\right) d \mu$ s.t. $v\left(x_{k}, \delta x_{k}, k\right) \geq$ $\underline{m}\left\|\xi_{1, k}-\xi_{2, k}\right\|_{2}^{2}$. Then the mean squared distance between the two trajectories of the system (12) satisfies the bound

$$
E_{\varepsilon_{0}}\left[\left\|\xi_{1, k}-\xi_{2, k}\right\|^{2}\right] \leq \frac{1}{\underline{m}}\left(\tilde{\gamma}_{d}^{k} v_{0}+C_{d} \frac{1-\tilde{\gamma}_{d}^{k}}{1-\tilde{\gamma}_{d}}\right)
$$

where $\tilde{\gamma}_{d}=1-\gamma_{2} \in(0,1)$ and $\gamma_{2} \in(0,1)$ is a positive constant that satisfies $1-\gamma_{2} \geq(\bar{m} / \underline{m})\left(1-\gamma_{d}\right)$ with $\gamma_{d}$ denoting the contraction rate for the deterministic system (8). The subscript $\varepsilon_{0}$ means that $x_{0}, \delta x_{0}$, and $t_{0}$ are fixed.

Proof: Let $v_{k}=v_{k}\left(x_{k}, \delta x_{k}, k\right)$ and $M_{k}=M_{k}\left(x_{k}, k\right)$ for notation simplicity. Using the bounds and the incremental system (12), we have that

$$
\begin{aligned}
& v_{k+1} \leq \bar{m} \int_{0}^{1}\left\|\frac{\partial f_{k}}{\partial x_{k}} \frac{\partial x_{k}}{\partial \mu}+\frac{\partial G_{k}}{\partial \mu} w_{k}\right\|^{2} d \mu \\
\leq & \frac{\bar{m}}{\underline{m}}\left(1-\gamma_{d}\right) \int_{0}^{1} \frac{\partial x_{k}^{T}}{\partial \mu} M_{k} \frac{\partial x_{k}}{\partial \mu} d \mu \\
& +\bar{m} \int_{0}^{1}\left(2 \frac{\partial x_{k}^{T}}{\partial \mu} \frac{\partial f_{k}^{T}}{\partial x_{k}} \frac{\partial G_{k}}{\partial \mu} w_{k}+w_{k}^{T} \frac{\partial G_{k}^{T}}{\partial \mu} \frac{\partial G_{k}}{\partial \mu} w_{k}\right) d \mu
\end{aligned}
$$

where $f_{k}=f_{k}\left(x_{k}, k\right)$ and $G_{k}=G_{k}\left(x_{k}, k\right), i=1,2$ for notation simplicity. Taking the conditional expected value of (14) when $x_{k}, \delta x_{k}$, and $k$ are fixed, we have that

$$
\begin{aligned}
& E_{\varepsilon_{k}}\left[v_{k+1}\right] \leq \gamma_{m} v_{k}+\bar{m} E_{\varepsilon_{k}}\left[\int_{0}^{1} w_{k}^{T} \frac{\partial G_{k}}{\partial \mu} \frac{\partial G_{k}}{\partial \mu} w_{k} d \mu\right] \\
& \leq \gamma_{m} v_{k}+\bar{m} E_{\varepsilon_{k}}\left[\operatorname{Tr}\left(w_{1, k} w_{1, k}^{T} G_{1, k}^{T} G_{1, k}\right)\right] \\
& \quad+\bar{m} E_{\varepsilon_{k}}\left[\operatorname{Tr}\left(w_{2, k} w_{2, k}^{T} G_{2, k}^{T} G_{2, k}\right)\right] \\
& \leq \gamma_{m} v_{k}+\bar{m}\left(\operatorname{Tr}\left(G_{1, k}^{T} G_{1, k}\right)+\operatorname{Tr}\left(G_{2, k}^{T} G_{2, k}\right)\right) .
\end{aligned}
$$

where $\gamma_{m}=\bar{m} / \underline{m}\left(1-\gamma_{d}\right)$ and $x_{k}, \delta x_{k}$, and $k$ are denoted as $\varepsilon_{k}$. Suppose that there exists $\gamma_{2} \in(0,1)$ s.t. $\gamma_{m} \leq 1-\gamma_{2}$. Using the property $E_{\varepsilon_{k-2}}\left[v_{k}\right]=E_{\varepsilon_{k-2}}\left[E_{\varepsilon_{k-1}}\left[v_{k}\right]\right]$, we have that

$$
E_{\varepsilon_{k-2}}\left[v_{k}\right] \leq \tilde{\gamma}_{d}^{2} v_{k-2}+C_{d}+C_{d} \tilde{\gamma}_{d}
$$

where $\tilde{\gamma}_{d}=1-\gamma_{2}$. Continuing this operation yields

$$
\begin{aligned}
\underline{m} E_{\varepsilon_{0}}\left[\left\|\xi_{1, k}-\xi_{2, k}\right\|^{2}\right] & \leq \tilde{\gamma}_{d}^{k} v_{0}+C_{d} \sum_{i=0}^{k-1} \tilde{\gamma}_{d}^{i} \\
& =\tilde{\gamma}_{d}^{k} v_{0}+C_{d} \frac{1-\tilde{\gamma}_{d}^{k}}{1-\tilde{\gamma}_{d}}
\end{aligned}
$$

which gives (13) by dividing (17) by $\underline{m}$.

Remark 1: Since $\tilde{\gamma}_{d} \in(0,1)$, we have

$$
\lim _{k \rightarrow \infty} E_{\varepsilon_{0}}\left[\left\|\xi_{1, k}-\xi_{2, k}\right\|^{2}\right] \leq \frac{C_{d}}{\underline{m}\left(1-\tilde{\gamma}_{d}\right)}=\frac{C_{d}}{\underline{m} \gamma_{2}} .
$$




\section{Stabilizing Stochastic Feedback Controller}

This section presents an exponentially stabilizing feedback controller for Itô stochastic nonlinear systems, which will be optimized in Sec. IV. Note that this design is not for finding an optimal control trajectory, which can be used as desired values in the present control design. Also, we focus on a controller design for continuous-time systems from this section, although this approach can readily be used for discrete-time and hybrid systems using Theorem 1 presented earlier in Sec. II.

\section{A. Problem Formulation}

Consider the following input-affine Itô stochastic nonlinear system

$$
\begin{aligned}
d x & =f(x, t) d t+B(x, t) u d t+G_{1}(x, t) d W_{1} \\
d x_{d} & =f\left(x_{d}, t\right) d t+B\left(x_{d}, t\right) u_{d} d t .
\end{aligned}
$$

where $x_{d}$ and $u_{d}$ are the desired trajectory and desired input respectively, which are deterministic as they are assumed to be given.

Remark 2: Since $\dot{x}_{d}-f\left(x_{d}, t\right) \in \operatorname{Im} B\left(x_{d}, t\right)$ must hold for a feasible desired trajectory, $u_{d}$ can be obtained as $u_{d}=B\left(x_{d}, t\right)^{+}\left(\dot{x}_{d}-f\left(x_{d}, t\right)\right)$ where $(\cdot)^{+}$denotes the MoorePenrose inverse. When $\operatorname{Ker} B\left(x_{d}, t\right)=\{0\}$, then $u_{d}$ is the unique solution to $B\left(x_{d}, t\right) u_{d}=\dot{x}_{d}-f\left(x_{d}, t\right)$. When $\operatorname{Ker} B\left(x_{d}, t\right) \neq\{0\}$, then $u_{d}$ is a solution with the smallest Euclidean norm. Also, $u_{d}$ can be found by solving some optimal control problem [39]. Further, general systems with $\dot{x}=f(x, u)$ can be transformed into an input-affine form by treating $\dot{u}$ as another input.

In SDC form, (19) and (20) can be expressed as

$$
\begin{aligned}
d x & =A(\rho, x, t) x d t+B(x, t) u d t+G_{1}(x, t) d W_{1} \\
d x_{d} & =A\left(\rho, x_{d}, t\right) x_{d} d t+B\left(x_{d}, t\right) u_{d} d t
\end{aligned}
$$

where $\rho=\left(\rho_{1}, \cdots, \rho_{s_{1}}\right)$ are the coefficients of the convex combination of SDC parameterizations, i.e., $A(\rho, x, t)=$ $\sum_{i=1}^{s_{1}} \rho_{i} A_{i}(x, t)$. Writing the system dynamics (19) in SDC form provides a design flexibility to mitigate effects of stochastic noise while verifying that the system is controllable as shall be seen later on.

Remark 3: Let $\Omega$ be the state set that is a bounded open subset of some Euclidean space that contains the origin, i.e., $0 \in \Omega \subseteq \mathbb{R}^{n}$. Under the assumptions $f(0)=0$ and $f(x)$ is a continuously differentiable function of $x$ on $\Omega$, there always exists at least one continuous nonlinear matrix-valued function $A(x)$ on $\Omega$ s.t. $f(x)=A(x) x$, where $A: \Omega \rightarrow \mathbb{R}^{n \times n}$ is found by mathematical factorization and is nonunique when $n>1[11]$.

\section{B. Stabilizing Feedback Controller Design}

A nonlinear feedback tracking controller is designed as

$$
\begin{aligned}
u & =-K(x, t)\left(x-x_{d}\right)+u_{d} \\
& =-R^{-1}(x, t) B^{T}(x, t) P(x, t)\left(x-x_{d}\right)+u_{d}
\end{aligned}
$$

where $P(x, t)$ is a positive definite matrix which satisfies the following equation

$$
\begin{aligned}
-\dot{P}(x, t)= & A^{T}(\rho, x, t) P(x, t)+P(x, t) A(\rho, x, t) \\
& +2 \alpha P(x, t)+2 \kappa P^{2}(x, t) \\
& -P(x, t) B(x, t) R^{-1}(x, t) B^{T}(x, t) P(x, t) .
\end{aligned}
$$

Define $A_{c l}(\rho, q, t), \Delta A(\rho, q, t)$, and $\Delta B(q, t)$ as

$$
\begin{aligned}
A_{c l}(\rho, q, t) & =A\left(\rho, q+x_{d}, t\right)-B\left(q+x_{d}, t\right) K\left(q+x_{d}, t\right) \\
\Delta A(\rho, q, t) & =A\left(\rho, q+x_{d}, t\right)-A\left(\rho, x_{d}, t\right) \\
\Delta B(q, t) & =B\left(q+x_{d}, t\right)-B\left(x_{d}, t\right) .
\end{aligned}
$$

Substituting (23) into (21) yields

$$
d e=f_{e}(e, t) d t+G_{1}\left(e+x_{d}, t\right) d W_{1}
$$

where $e=x-x_{d}$ and

$$
f_{e}(e, t)=A_{c l}(\rho, e, t) e+\Delta A(\rho, e, t) x_{d}+\Delta B(e, t) u_{d} .
$$

Let us define a virtual system for the deterministic system

$$
\begin{aligned}
\dot{q} & =A_{c l}(\rho, e, t) q+\Delta A(\rho, q, t) x_{d}+\Delta B(q, t) u_{d} \\
& =f_{v}(q, t) .
\end{aligned}
$$

Note that (28) has $e$ and 0 as particular solutions. The virtual dynamics of (28) is expressed as

$$
\delta \dot{q}=A_{c l}(\rho, e, t) \delta q+\frac{\partial\left(\Delta A x_{d}+\Delta B u_{d}\right)}{\partial q} \delta q .
$$

Using $f_{v}(q, t)$, the virtual system for (26) with respect $q$ is defined as

$$
d q=f_{v}(q(\mu, t), t) d t+G(q(\mu, t), t) d W
$$

where $\mu \in[0,1]$ is introduced to parameterize the trajectories $q=e$ and $q=0$, i.e., $q(\mu=0, t)=e, q(\mu=1, t)=0$, $G(q(0, t), t)=G_{1}\left(e+x_{d}, t\right)$, and $G(q(1, t), t)=0_{n \times d}$.

Remark 4: (30) has $q=e$ and $q=0$ as particular solutions.

- $f_{v}(e, t)=f_{e}(e, t)$ and $G(e, t)=G_{1}\left(e+x_{d}, t\right)$ when $q=e$.

- $f_{v}(0, t)=\Delta A(\rho, 0, t) x_{d}+\Delta B(0, t) u_{d}=0$ and $G(0, t)=$ $0_{n \times d}$ when $q=0$.

Before analyzing the stability of the system with this controller, let us introduce the following assumption.

Assumption 1: There exist positive constants $p, \bar{p}, \bar{p}_{x}, \bar{p}_{x^{2}}$, $\underline{r}, \bar{r}, \delta_{1}, \delta_{2}, \beta$, and $g_{1}$ s.t. $p \leq\|P(x, t)\| \leq \bar{p}, \underline{r} \leq\|R(x, t)\| \leq$ $\bar{r}, \bar{p}_{x}=\sup _{t \geq 0, i, j}\left\|\left(p_{i j}\right)_{x}\right\|, \bar{p}_{x^{2}}=\sup _{t \geq 0, i, j}\left\|\partial^{2}\left(p_{i j}\right) / \partial x^{2}\right\|$, $\left\|\partial\left(\Delta A x_{d}\right) / \partial q\right\| \leq \delta_{1},\left\|\partial\left(\Delta B u_{d}\right) / \partial q\right\| \leq \delta_{2}, \beta \leq\|B(x, t)\|$, and $\left\|G_{1}(x, t)\right\|_{F} \leq g_{1}, \forall \rho, x, t$, where $p_{i j}$ is the $(i, j)$ component of $P(x, t)$

Now, we introduce the following theorem that guarantees the exponential stability of the controller (23).

Theorem 2: Suppose that Assumption 1 is satisfied and that there exists $\alpha_{1}>0$ s.t.

$$
\begin{aligned}
\left(\alpha-\alpha_{1}\right) \underline{p} & \geq \kappa_{1} \bar{p}-\kappa_{2} \underline{p}^{2}-\kappa \underline{p}^{2}+\alpha_{g} \\
\alpha-\alpha_{1} & \geq 0
\end{aligned}
$$

where $2 \alpha_{g}=g_{1}^{2}\left(\bar{p}_{x} \varepsilon+\bar{p}_{x^{2}} / 2\right)$ with $\varepsilon$ being an arbitrary positive constant. Then the mean squared distance between 
the trajectory of the system with the controller (23) and that of the desired system is exponentially bounded with the bound

$$
E\left[\left\|x_{d}-x\right\|^{2}\right] \leq \frac{1}{\underline{p}}\left(E[V(x(0), \delta q(0), 0)] e^{-2 \alpha_{1} t}+\frac{C}{2 \alpha_{1}}\right)
$$

where $V(x, \delta q, t)=\int_{0}^{1}(\partial q / \partial \mu)^{T} P(x, t)(\partial q / \partial \mu) d \mu$ and $C=\bar{p} g_{1}^{2}+\left(\bar{p}_{x} g_{1}^{2}\right) / \varepsilon$.

Proof: For notation simplicity, let $P=P(x, t), A=$ $A(\rho, x, t), B=B(x, t), R=R(x, t), G=G(q, t)$, and $\phi=$ $\phi(\rho, q, t)=\partial\left(\Delta A x_{d}\right) / \partial q+\partial\left(\Delta B u_{d}\right) / \partial q$. Let us define an infinitesimal differential generator as

$$
\begin{aligned}
\mathscr{L}[V(x, \delta q, t)]= & \frac{\partial V}{\partial t}+\sum_{i=1}^{n}\left(\frac{\partial V}{\partial x_{i}} f_{i}+\frac{\partial V}{\partial\left(\delta q_{i}\right)} \frac{\partial f_{v}}{\partial q} \delta q\right) \\
& +\frac{1}{2} \sum_{i=1}^{n} \sum_{j=1}^{n}\left[\frac{\partial^{2} V}{\partial x_{i} \partial x_{j}}\left(G_{1}(x, t) G_{1}^{T}(x, t)\right)_{i j}\right. \\
& +2 \frac{\partial^{2} V}{\partial x_{i} \partial\left(\delta q_{j}\right)}\left(G_{1}(x, t) \delta G^{T}(q, t)\right)_{i j} \\
& \left.+\frac{\partial^{2} V}{\partial\left(\delta q_{i}\right)\left(\delta q_{j}\right)}\left(\delta G(q, t) \delta G^{T}(q, t)\right)_{i j}\right]
\end{aligned}
$$

where $f_{i}$ is the $i$ th component of $f$. Substituting (24), (30), and $V$ into (34) yields

$$
\begin{aligned}
\mathscr{L} V= & \int_{0}^{1} \frac{\partial q^{T}}{\partial \mu}\left(\dot{P}+A^{T} P+P A-2 P B^{T} R^{-1} B^{T} P\right. \\
& \left.+\phi^{T} P+P \phi\right) \frac{\partial q}{\partial \mu} d \mu+V_{2} \\
= & \int_{0}^{1} \frac{\partial q^{T}}{\partial \mu}\left(-2 \alpha P-2 \kappa P^{2}-P B^{T} R^{-1} B^{T} P\right. \\
& \left.+\phi^{T} P+P \phi\right) \frac{\partial q}{\partial \mu} d \mu+V_{2} .
\end{aligned}
$$

where $V_{2}$ is upper bounded by $\bar{V}_{2}$ [29] defined as

$$
V_{2} \leq \bar{V}_{2}=2 \alpha_{g} \int_{0}^{1}\left\|\frac{\partial q}{\partial \mu}\right\|^{2} d \mu+C .
$$

Under Assumption 1, we can compute the following bounds

$$
\|P \phi+\phi P\| \leq 2 \bar{p} \kappa_{1}, \quad\left\|P B^{T} R^{-1} B^{T} P\right\| \geq 2 \underline{p}^{2} \kappa_{2}
$$

where fixed constants $\kappa_{1}$ and $\kappa_{2}$ are defined as

$$
\kappa_{1}=\delta_{1}+\delta_{2}, \quad \kappa_{2}=\frac{\beta^{2}}{2 \bar{r}} .
$$

Applying (31) and (36) to (35), we have that

$$
\begin{aligned}
\mathscr{L} V & \leq 2 \int_{0}^{1} \frac{\partial q^{T}}{\partial \mu}\left(-\alpha P+\left(\kappa_{1} \bar{p}-\kappa_{2} \underline{p}^{2}-\kappa \underline{p}^{2}\right) I\right) \frac{\partial q}{\partial \mu} d \mu+V_{2} \\
& \leq 2 \int_{0}^{1} \frac{\partial q^{T}}{\partial \mu}\left(-\alpha P+\left(\alpha-\alpha_{1}\right) \underline{p I}\right) \frac{\partial q}{\partial \mu} d \mu+C
\end{aligned}
$$

Then the condition (32) implies that

$$
\mathscr{L} V \leq-2 \alpha_{1} V(x, \delta q, t)+C .
$$

Therefore, (40) along with Lemma 2 completes the derivation of (33).

\section{Main Result: Stochastic Optimal Feedback CONTROLLER}

The state feedback controller (23) is only a stabilizing controller as we proved in Theorem 2. To make this controller optimal in some sense, we formulate an optimization problem which minimizes an upper bound of the steady-state mean squared distance in (33) to find the optimal controller parameters, i.e.,

$$
\begin{aligned}
& \operatorname{minimize} \frac{\bar{p}}{\frac{p}{p}}+c_{1} \frac{1}{p} \\
& \text { subject to }(24),(31), \text { and }(32)
\end{aligned}
$$

where $c_{1}=\bar{p}_{x} / \varepsilon$ and we assume that $g_{1}$ and $\alpha_{1}$ are given. We propose one way to reformulate this non-convex optimization problem as a convex optimization problem.

\section{A. Constraint (24)}

The constraint (24) can be relaxed to the following matrix inequality

$$
\begin{aligned}
& A^{T}(\rho, x, t) P(x, t)+P(x, t) A(\rho, x, t)+2 \alpha P(x, t) \\
& +2 \kappa P^{2}(x, t)-P(x, t) B(x, t) R^{-1}(x, t) B^{T}(x, t) P(x, t) \preceq 0 .
\end{aligned}
$$

Multiplying it by $Q(x, t)=P^{-1}(x, t)$ from both sides, (42) is equivalent to

$$
\begin{aligned}
& Q(x, t) A^{T}(\rho, x, t)+A(\rho, x, t) Q(x, t)+2 \alpha Q(x, t) \\
& +2 \kappa I-B(x, t) R^{-1}(x, t) B^{T}(x, t) \preceq 0 .
\end{aligned}
$$

Proposition 1: The bilinear matrix inequality (43) can be converted to the following linear matrix inequality (LMI) by Shor's relaxation in terms of variables $Q, Q_{\rho_{i}}=\rho_{i} Q, \rho_{i}$, and $\kappa$, i.e.

$$
\sum_{i=1}^{s_{1}} A_{i} Q_{\rho_{i}}+\sum_{i=1}^{s_{1}} Q_{\rho_{i}} A_{i}^{T}+2 \alpha Q+2 \kappa I-B R^{-1} B^{T} \preceq 0
$$

with the following constraints to ensure controllability and $Q_{\rho_{i}}=\rho_{i} Q$

$$
\begin{aligned}
& Q \succ 0, Q_{\rho_{i}} \succ 0, \sum_{i=1}^{s_{1}} Q_{\rho_{i}}=Q, \operatorname{sym}\left[\begin{array}{cc}
I & Q \\
\rho_{i} I & Q_{\rho_{i}}
\end{array}\right] \succeq 0, \quad(45) \\
& \sum_{i=1}^{s_{1}} \rho_{i}=1, \rho_{i} \in[0,1], c c_{k}(\rho, x)<0, \forall i, \forall k=1, \cdots, n_{c}
\end{aligned}
$$

where $\operatorname{sym}(\cdot)$ is a symmetric part of a matrix and $c c_{j}(\rho, x)<$ $0, \forall k=1, \cdots, n_{c}$ denotes $n_{c}$ number of convex constraints to maintain the controllability of the pair $(A, B)$. Then (44) and (45) are convex constraints in terms of decision variables assuming $\alpha$ is given.

$$
\text { Proof: See [29]. }
$$

\section{B. Objective Function and Contraction Condition}

Let us introduce a new variable $\chi$ defined as $\chi=\bar{p} / \underline{p}$ with additional constraint [33]

$$
\frac{1}{\bar{p}} I \preceq Q \preceq \frac{1}{\underline{p}} I .
$$


Using $\chi$, the objective function of (41) is upper bounded as follows

$$
\frac{\bar{p}}{\underline{p}}+c_{1} \frac{1}{\underline{p}}=\frac{\bar{p}}{\underline{p}}+c_{1} \frac{\chi}{\bar{p}} \leq \chi+c_{1} \frac{\chi^{2}}{\bar{p}}
$$

where $\chi \leq \chi^{2}$ is used to obtain the inequality. Defining additional variables $v=\bar{p},(\tilde{\cdot})=(\cdot) \bar{p},(46)$ is expressed as

$$
I \preceq \tilde{Q} \preceq \chi I .
$$

The constraints (44) and (45) can also be written in terms of the new variables

$$
\sum_{i=1}^{s_{1}} A_{i} \tilde{Q}_{\rho_{i}}+\sum_{i=1}^{s_{1}} \tilde{Q}_{\rho_{i}} A_{i}^{T}+2 \alpha \tilde{Q}+2 \tilde{\kappa} I-v B R^{-1} B^{T} \preceq 0
$$

where

$$
\begin{aligned}
& \tilde{Q} \succ 0, \tilde{Q}_{\rho_{i}} \succ 0, \sum_{i=1}^{s_{1}} \tilde{Q}_{\rho_{i}}=\tilde{Q}, \operatorname{sym}\left[\begin{array}{cc}
v I & \tilde{Q} \\
\tilde{\rho}_{i} I & \tilde{Q}_{\rho_{i}}
\end{array}\right] \succeq 0, \quad(50) \\
& \sum_{i=1}^{s_{1}} \tilde{\rho}_{i}=v, \tilde{\rho}_{i} \in[0, v], c c_{k}(\tilde{\rho}, x)<0, \quad \forall i, \quad \forall k=1, \cdots, n_{c} .
\end{aligned}
$$

Finally, multiplying (31) by $\bar{p} / p^{2}$, we have $\left(\alpha-\alpha_{1}\right)(\bar{p} / p) \geq$ $\kappa_{1}(\bar{p} / \underline{p})^{2}-\kappa_{2} \bar{p}-\kappa \bar{p}+\alpha_{g}\left(\bar{p} / \underline{p}^{2}\right)$. In terms of the new variables, this can be written as

$$
\left(\alpha-\alpha_{1}\right) \chi \geq \kappa_{1} \chi^{2}-\kappa_{2} v-\tilde{\kappa}+\alpha_{g} \frac{\chi^{2}}{v} .
$$

Assuming that $\alpha, \alpha_{1}, \kappa_{1}, \kappa_{2}$, and $\alpha_{g}$ are given, this is a convex constraint as $a^{2} / b$ is a convex function for $a$ and $b$ if $b>0$. As a result, the optimization problem (41) with the new objective function (47), which is an upper bound of the steady-state tracking error, reduces to

$$
\begin{aligned}
& \text { minimize } \chi+c_{1} \frac{\chi^{2}}{v} \\
& \text { subject to (48), (49), (50), and (51). }
\end{aligned}
$$

Note that $\chi+\left(c_{1} \chi^{2}\right) / v$ is a convex function in terms of decision variables $\chi$ and $v>0$ assuming $c_{1}$ is given.

Proposition 2: The optimization problem (52) is convex in terms of decision variables $\tilde{\kappa}>0, v>0, \chi>0, \tilde{Q} \succ 0$, $\tilde{Q}_{\rho_{i}} \succ 0$, and $\tilde{\rho}_{i} \in \mathbb{R}, \forall i=1, \cdots, s_{1} . \alpha, \alpha_{1} \geq 0$ s.t. $\alpha-\alpha_{1} \geq 0$ and $R(x, t) \succeq 0$ have to be specified by a user. The optimal solution provides an upper bound of the optimal steady-state tracking error.

Remark 5: (52) can be simplified further by selecting some $\rho$ that satisfies $\sum_{i} \rho_{i}=1$ and that preserves the controllability a priori. Then, the constraints (49) and (50) are reduced to one LMI constraint.

\section{Variations of Convex Optimization Problem}

1) Penalty Formulation: Although (52) is convex, the constraint (51) can be too conservative for the problem to be feasible because of the fact that we consider an upper bound of the steady-state tracking error. This motivate us to introduce a penalty formulation with a new decision variable $\zeta$ that satisfies the following inequalities

$$
\begin{aligned}
& \zeta \geq-\left(\alpha-\alpha_{1}\right) \chi+\kappa_{1} \chi^{2}-\kappa_{2} v-\tilde{\kappa}+\alpha_{g} \frac{\chi^{2}}{v} \\
& \zeta \geq 0 .
\end{aligned}
$$

The penalty formulation of (52) is given as

$$
\begin{aligned}
& \text { minimize } \chi+c_{1} \frac{\chi^{2}}{v}+\zeta \\
& \text { subject to (48), (49), (50), (53) and (54). }
\end{aligned}
$$

2) Input Constraints through the norm of $K(x, t)$ : Let us consider the case when the input constraint can be relaxed to a constraint $\left\|u_{s}\right\| \leq u_{\max }$. Then the sufficient condition for the relaxed input constraint is

$$
v\left\|R^{-1} B^{T}\right\|\|s\| \leq u_{\max } \lambda_{\min }(\tilde{Q}), \forall t, x
$$

which is a convex constraint that can be implemented in (52) without losing their convexity.

\section{Summary for Stochastic Optimal Feedback Controller}

The stochastic optimal feedback controller design is summarized as follows.

Proposition 3: An optimal stochastic nonlinear feedback controller is designed as (23), where $P(x, t)$ is selected by the convex optimization problem (52) or (55) with decision variables $\tilde{\kappa} \in \mathbb{R}, v \in \mathbb{R}, \chi \in \mathbb{R}, \zeta \in \mathbb{R}, \tilde{Q} \in \mathbb{R}^{n \times n}, \tilde{Q}_{\rho_{i}} \in \mathbb{R}^{n \times n}$, and $\tilde{\rho}_{i} \in \mathbb{R}, \forall i=1, \cdots, s_{1}$.

Remark 6: The constraints are state- and time-dependent, which means that (52) or (55) has to be solved at each time instant.

This controller synthesis algorithm provides a convex optimization-based methodology for selecting decision variables that minimize an upper bound of the steady-state tracking error. The controllability constraint at each step can be expressed in a simple form thanks to the SDC parametrization of the original dynamics. The convexity of the optimization problem (52) or (55) allows us to solve this problem using computationally-efficient numerical techniques such as the polynomial-time interior point methods [33]-[35]. In practice, the solution to the state-dependent algebraic Riccati equation can also be obtained efficiently by using various numerical techniques [40], [41].

\section{NumERicAl Simulation}

The performance of the controller in Proposition 3 is evaluated using the following spacecraft attitude control example.

\section{A. Simulation Setup}

We consider the spacecraft attitude dynamics given in [42] with stochastic noise. The simulation parameters are selected as $J=\left[\begin{array}{ccc}150 & 0 & -100 \\ 0 & 270 & 0 \\ -100 & 0 & 300\end{array}\right], q(0)=\left[\begin{array}{ll}0.9 & -\end{array}\right.$ $\left.\begin{array}{ll}0.9 & 0.7\end{array}\right]^{T}, \dot{q}(0)=\left[\begin{array}{lll}0.6 & 0.7 & -0.5\end{array}\right]^{T}$, and $G(x, t)=0.2 \times \mathbf{1}_{n \times d}$ with $d=1$. The desired trajectories are defined as $q_{1 d}=$ 

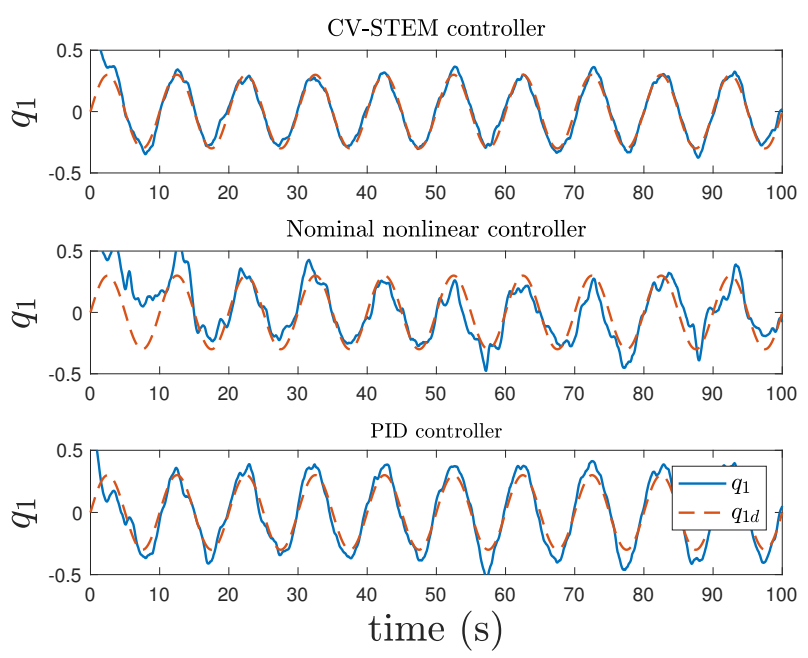

Fig. 1: Modified Rodrigues parameter $q_{1}$
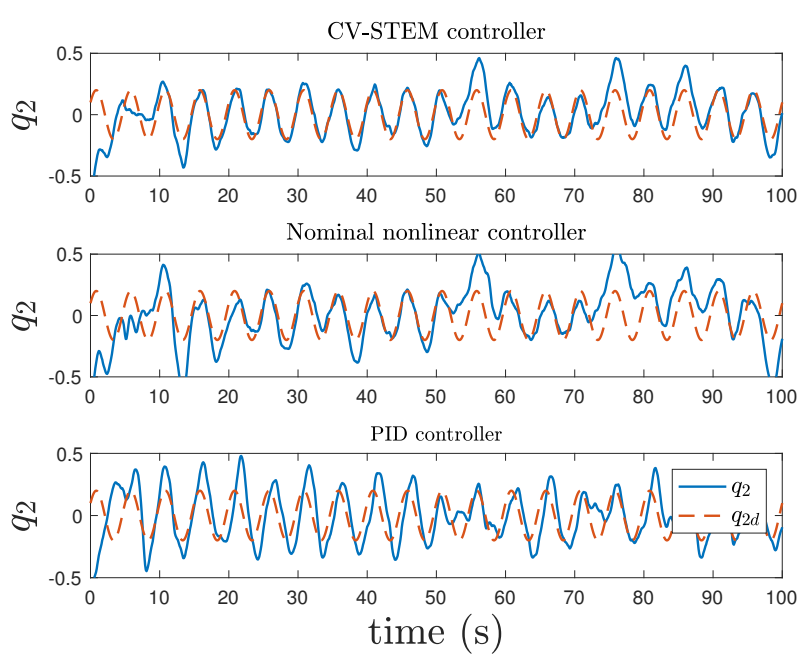

Fig. 2: Modified Rodrigues parameter $q_{2}$
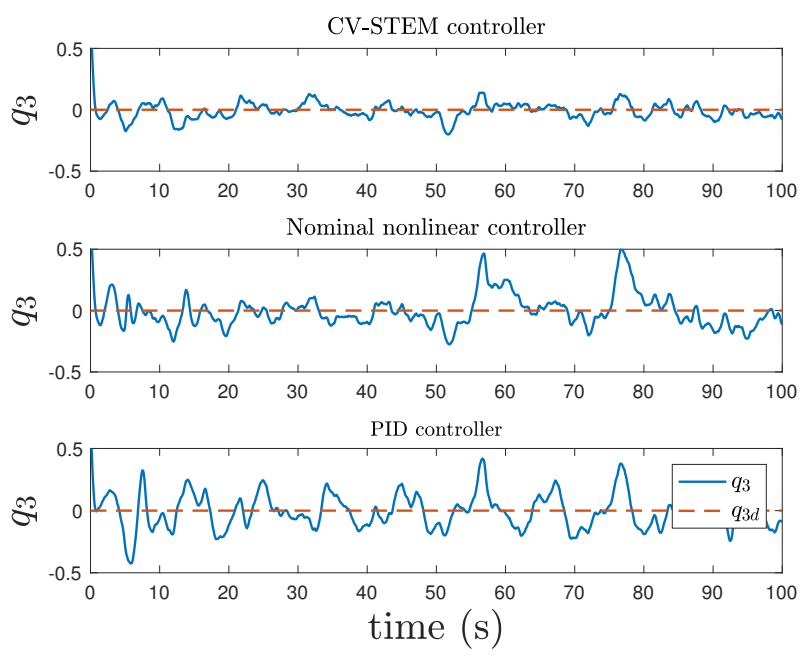

Fig. 3: Modified Rodrigues parameter $q_{3}$
$0.3 \sin (2 \pi(0.1) t), q_{2 d}=0.2 \sin (2 \pi(0.2) t+\pi / 6)$, and $q_{3 d}=0$. Parameters of the proposed controller are updated at every time instant by solving the convex optimization problem using the $c v x$ toolbox in Matlab [43], [44]. Coefficients of the convex combination of SDC matrices are selected a priori so that they preserve controllability.

For comparison, the same simulation is performed for a PID controller and a nominal nonlinear controller [36] that guarantees exponential stability. The $\mathrm{P}, \mathrm{I}$ and $\mathrm{D}$ gains are selected as $K_{p}=500 I, K_{i}=300 I, K_{d}=500 I$ respectively. $K_{r}=100 I$ and $\Lambda=1$ are used for the controller [36].

Remark 7: The parameters for each controller are selected to achieve similar control effort for each controller to ensure a fair comparison.

\section{B. Simulation Results}

Figures 1, 2, and 3 show the comparison between the controlled and the desired modified Rodorigues parameters $q$ for the proposed controller in Proposition 3, a controller [36], and a PID controller. Figure 4 shows the steady-state tracking error and the control effort for each controller, which are computed by $\left\|x-x_{d}\right\|_{2}^{2}$ and $\int_{0}^{T}\|u\|_{2}^{2} d t$ where $x=[q \dot{q}]^{T}$. Note that $\left\|x-x_{d}\right\|_{2}^{2}$ as $t$ goes to infinity is the value we attempt to minimize. In order to improve the visibility, the simulation data of the tracking error is smoothed using a moving average filter which takes the average of every 150 consecutive samples at each time instant. It is shown that the controller in Proposition 3 achieves the smaller steady-state tracking error than that of [36] and PID with smaller amount of control effort as can be seen in Fig. 4.

\section{CONCLUSION}

In this paper, we proposed a convex optimization-based feedback tracking control approach for Itô stochastic nonlinear systems written using multiple SDC parameterizations. A rigorous proof on stochastic incremental exponential stability is provided using contraction analysis. We formulated a convex program for obtaining the optimal feedback gain and controller parameters, which minimize an upper bound of the steady-state tracking error. It is shown in the simulation that this controller outperforms a known exponentially-stabilizing nonlinear controller and a PID controller. The SDC parameterization of the original dynamics allows us to guarantee the controllability at each time instant through a simple constraint. We also derived discrete-time stochastic incremental contraction analysis for state- and time-dependent metrics,
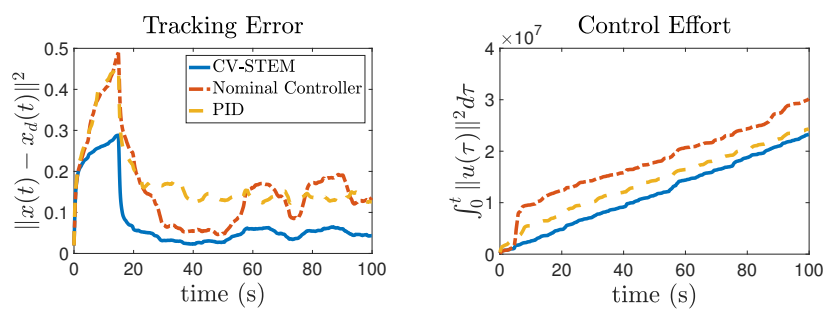

Fig. 4: Smoothed tracking error and control effort 
which can be useful in proving the stability of discretetime and hybrid stochastic nonlinear systems. This theorem implies a similar SDC-based design approach to nonlinear optimal feedback controllers for discrete-time stochastic nonlinear systems.

\section{ACKNOWLEDGEMENT}

This work was in part funded by the Jet Propulsion Laboratory, California Institute of Technology and Raytheon Company.

\section{REFERENCES}

[1] H. J. Kushner, Stochastic Stability and Control. Academic Press New York, 1967.

[2] V. Palleschi, F. Sarri, G. Marcozzi, and M. Torquati, "Numerical solution of the Fokker-Planck equation: A fast and accurate algorithm," Physics Lett. A, vol. 146, no. 7, pp. 378 - 386, 1990.

[3] A. Isidori, Nonlinear Control Systems. Berlin, Heidelberg: SpringerVerlag, 3rd ed., 1995.

[4] J.-J. E. Slotine and W. Li, Applied Nonlinear Control. Upper Saddle River, NJ: Pearson, 1991.

[5] H. K. Khalil, Nonlinear Systems. Upper Saddle River, NJ: PrenticeHall, 3rd ed., 2002.

[6] E. Sontag, "A Lyapunov-like characterization of asymptotic controllability," SIAM J. Control and Optimization, vol. 21, no. 3, pp. 462-471, 1983.

[7] E. D. Sontag, "A 'universal' construction of Artstein's theorem on nonlinear stabilization," Syst. Control Lett., vol. 13, no. 2, pp. 117 123, 1989.

[8] S. Prajna, A. Papachristodoulou, and F. Wu, "Nonlinear control synthesis by sum of squares optimization: A Lyapunov-based approach," in Proc. Asian Control Conf., vol. 1, pp. 157-165, July 2004.

[9] E. M. Aylward, P. A. Parrilo, and J.-J. E. Slotine, "Stability and robustness analysis of nonlinear systems via contraction metrics and SOS programming," Automatica, vol. 44, no. 8, pp. 2163 - 2170, 2008.

[10] J. R. Cloutier, "State-dependent Riccati equation techniques: An overview," in Proc. Amer. Control Conf., vol. 2, pp. 932-936, June 1997.

[11] T. Çimen, "Survey of state-dependent Riccati equation in nonlinear optimal feedback control synthesis," J. Guidance, Control, and Dynamics, vol. 35, pp. 1025-1047, July 2012.

[12] P. Florchinger, "Lyapunov-like techniques for stochastic stability," in Proc. IEEE Conf. Decis. Control, vol. 2, pp. 1145-1150, Dec. 1994.

[13] H. Deng, M. Krstic, and R. J. Williams, "Stabilization of stochastic nonlinear systems driven by noise of unknown covariance," IEEE Trans. Autom. Control, vol. 46, pp. 1237-1253, Aug. 2001.

[14] H. Deng and M. Krstic, "Stochastic nonlinear stabilization - I: A backstepping design," Syst. Control Lett., vol. 32, no. 3, pp. 143 $150,1997$.

[15] H. Deng and M. Krstic, "Output-feedback stochastic nonlinear stabilization," IEEE Trans. Autom. Control, vol. 44, pp. 328-333, Feb. 1999.

[16] W. Q. Zhu, "Nonlinear stochastic dynamics and control in Hamiltonian formulation," Applied Mechanics Reviews, vol. 59, pp. 230-248, July 2006.

[17] W. Zhang and B. Chen, "State feedback $\mathscr{H}_{\infty}$ control for a class of nonlinear stochastic systems," SIAM J. Control and Optimization, vol. 44, no. 6, pp. 1973-1991, 2006.

[18] S. Peng, "A general stochastic maximum principle for optimal control problems," SIAM J. Control and Optimization, vol. 28, no. 4, pp. 966979, 1990.

[19] E. Todorov and W. Li, "A generalized iterative LQG method for locally-optimal feedback control of constrained nonlinear stochastic systems," in Proc. Amer. Control Conf., vol. 1, pp. 300-306, June 2005.

[20] D. P. Bertsekas, Dynamic Programming and Optimal Control. Athena Scientific, 2nd ed., 2000.

[21] E. Tse, Y. Bar-Shalom, and L. Meier, "Wide-sense adaptive dual control for nonlinear stochastic systems," IEEE Trans. Autom. Control, vol. 18, pp. 98-108, Apr. 1973.
[22] D. S. Bayard, "A forward method for optimal stochastic nonlinear and adaptive control," IEEE Trans. Autom. Control, vol. 36, pp. 10461053, Sept. 1991

[23] A. Mesbah, "Stochastic model predictive control: An overview and perspectives for future research," IEEE Control Systems Mag., vol. 36, pp. 30-44, Dec. 2016.

[24] A. Mesbah, S. Streif, R. Findeisen, and R. D. Braatz, "Stochastic nonlinear model predictive control with probabilistic constraints," in Proc. Amer. Control Conf., pp. 2413-2419, June 2014.

[25] D. Angeli, "A Lyapunov approach to incremental stability properties,' IEEE Trans. Autom. Control, vol. 47, pp. 410-421, Mar. 2002.

[26] W. Lohmiller and J.-J. E. Slotine, "On contraction analysis for nonlinear systems," Automatica, vol. 34, no. 6, pp. 683 - 696, 1998.

[27] J. Jouffroy and J.-J. E. Slotine, "Methodological remarks on contraction theory," in IEEE Conf. Decis. Control, vol. 3, pp. 2537-2543, Dec. 2004.

[28] Q. Pham, N. Tabareau, and J.-J. E. Slotine, "A contraction theory approach to stochastic incremental stability," IEEE Trans. Autom. Control, vol. 54, pp. 816-820, Apr. 2009.

[29] A. P. Dani, S.-J. Chung, and S. Hutchinson, "Observer design for stochastic nonlinear systems via contraction-based incremental stability," IEEE Trans. Autom. Control, vol. 60, pp. 700-714, Mar. 2015.

[30] J.-J. E Slotine, W. Wang, and K. El Rifai, "Contraction analysis of synchronization in networks of nonlinearly coupled oscillators," in 16th Int. Symp. Mathematical Theory of Networks and Systems, July 2004.

[31] W. Lohmiller and J.-J. E. Slotine, "Nonlinear process control using contraction theory," AIChE Journal, vol. 46, pp. 588 - 596, Mar. 2000.

[32] Q. Pham, "Analysis of discrete and hybrid stochastic systems by nonlinear contraction theory," in 2008 10th Int. Conf. on Control, Automation, Robotics and Vision, pp. 1054-1059, Dec. 2008.

[33] S. Boyd, L. El Ghaoui, E. Feron, and V. Balakrishnan, Linear Matrix Inequalities in System and Control Theory, vol. 15 of Studies in Applied Mathematics. Philadelphia, PA: SIAM, June 1994.

[34] S. Boyd and L. Vandenberghe, Convex Optimization. Cambridge University Press, Mar. 2004.

[35] A. Ben-Tal and A. S. Nemirovskiaei, Lectures on Modern Convex Optimization: Analysis, Algorithms, and Engineering Applications. Philadelphia, PA, USA: SIAM, 2001.

[36] S. Bandyopadhyay, S.-J. Chung, and F. Y. Hadaegh, "Nonlinear attitude control of spacecraft with a large captured object," J. Guidance, Control, and Dynamics, vol. 39, pp. 754-769, Jan. 2016.

[37] S.-J. Chung, S. Bandyopadhyay, I. Chang, and F. Y. Hadaegh, "Phase synchronization control of complex networks of Lagrangian systems on adaptive digraphs," Automatica, vol. 49, no. 5, pp. $1148-1161$ 2013.

[38] T.-J. Tarn and Y. Rasis, "Observers for nonlinear stochastic systems," IEEE Trans. Autom. Control, vol. 21, pp. 441-448, Aug. 1976.

[39] D. E. Kirk, Optimal Control Theory: An Introduction. Dover Publications, Apr. 2004.

[40] E. B. Erdem and A. G. Alleyne, "Design of a class of nonlinear controllers via state dependent Riccati equations," IEEE Transactions on Control Systems Technology, vol. 12, pp. 133-137, Jan. 2004.

[41] A. Bogdanov and E. A. Wan, "State-dependent Riccati equation control for small autonomous helicopters," Journal of Guidance, Control, and Dynamics, vol. 30, pp. 47-60, Jan. 2007.

[42] S.-J. Chung, U. Ahsun, and J.-J. E. Slotine, "Application of synchronization to formation flying spacecraft: Lagrangian approach," $J$. Guidance, Control, and Dynamics, vol. 32, pp. 512-526, Mar. 2009.

[43] M. Grant and S. Boyd, "CVX: Matlab software for disciplined convex programming, version 2.1.” http://cvxr.com/cvx, Mar. 2014

[44] M. C. Grant and S. P. Boyd, "Graph implementations for nonsmooth convex programs," in Recent Advances in Learning and Control, pp. 95-110, Springer London, 2008. 